\title{
The effect of vitamin D supplementation on serum 25(OH)D concentration in Royal Navy submariners
}

\author{
F. Gunner ${ }^{1}$, M. Lindsay ${ }^{1}$, P.E.H. Brown ${ }^{1}$, A.M. Shaw ${ }^{1}$, S.E. Britland ${ }^{1}$, T. Davey ${ }^{1}$, \\ S.A. Lanham-New ${ }^{2}$, B.A. Griffin ${ }^{2}$ and J.L. Fallowfield ${ }^{1}$ \\ ${ }^{1}$ Environmental and Medicine Sciences, The Institute of Naval Medicine, Gosport, Hampshire, UK, PO12 2DL and \\ ${ }^{2}$ Department of Nutritional Sciences, School of Biosciences and Medicine, Faculty of Health and Medical Sciences, \\ University of Surrey, Guildford, Surrey, GU2 $7 X H$, UK
}

Royal Navy (RN) submariners routinely undergo periods of up to 3 months in an enclosed environment completely devoid of natural sunlight. They may therefore be at risk of low or deficient levels of circulating vitamin $\mathrm{D}$, and thus could benefit from supplementation. Information on the effects of prolonged submergence on vitamin D status and supplementation is limited. Furthermore comparison with existing literature is confounded by methodological differences. ${ }^{(1,2)}$ This study investigated serum $25(\mathrm{OH}) \mathrm{D}$ concentration (as a marker of vitamin D status) in deployed and non-deployed RN submariners.

Two cohorts of male submariners deploying in winter $(\mathrm{WIN} ; \mathrm{n}=32$ ) and summer (SUMM; $\mathrm{n}=64)$ for 12 weeks, and one cohort of male submariners who remained alongside for a similar duration $(H O M E ; n=34)$ volunteered for the study. Daily vitamin $D$ supplements $(2400 \mathrm{IU} ; \equiv 60 \mathrm{mg}$ ) were available via prescription for deployed cohorts only. Blood samples were drawn pre and post deployment and measurements of serum $25(\mathrm{OH}) \mathrm{D}$ were performed by liquid chromatography tandem mass spectrometry. The study was approved by the Ministry of Defence Research Ethics Committee and volunteers provided informed consent. Differences in serum $25(\mathrm{OH}) \mathrm{D}$ concentration were assessed by three-way mixed ANOVA (season, supplement use and time) and one-way ANOVA (predeployment concentration between groups).

Pre deployment serum $25(\mathrm{OH}) \mathrm{D}$ concentration was lower for WIN compared to SUMM (38 \pm 16 vs. $\left.53 \pm 20 \mathrm{nmol.1^{-1 }}, \mathrm{P}<0.001\right)$. It was higher at post deployment in volunteers who had supplemented with vitamin D compared with those who had not (mean difference of $47 \pm 4$ nmol. $1^{-1}, \mathrm{P}<0 \cdot 001$ ) (Fig. 1).

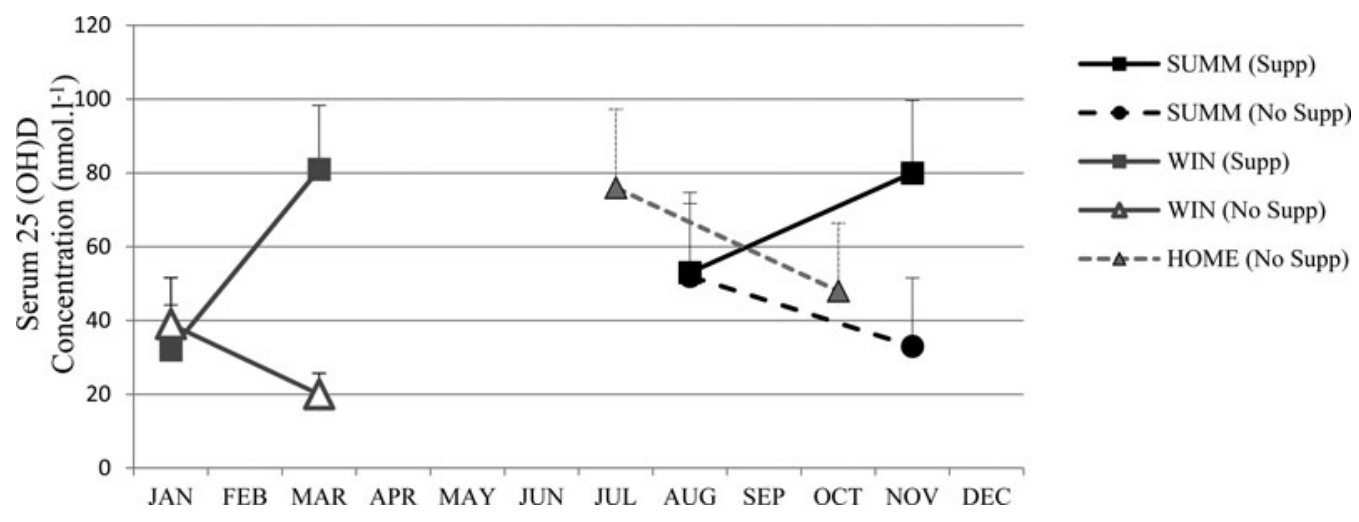

Fig. 1. Serum 25(OH)D Concentration in Supplement Users (Supp) and Non Supplement Users (No Supp)

This study reports decreases in serum $25(\mathrm{OH}) \mathrm{D}$ in both deployed and non-deployed RN submariners without vitamin D supplementation, irrespective of season but notably below $25 \mathrm{nmol} .1^{-1}$ in winter. Taking supplements is effective in preventing this decrease, but more research is required to determine the most practical and effective dose for this population given the likelihood of repeated deployment throughout the year.

1. Gasier et al. (2014) Calcified Tissue International 95(3): 229-39.

2. Holy et al. (2012) Journal of Applied Physiology 112, 587-596. 